\title{
Local rings of embedding codepth 3: A classification algorithm
}

\section{LARS WinTHER CHRISTENSEN AND OANA VELICHE}

\begin{abstract}
Let $I$ be an ideal of a regular local ring $Q$ with residue field $k$. The length of the minimal free resolution of $R=Q / I$ is called the codepth of $R$. If it is at most 3 , then the resolution carries the structure of a differential graded algebra, and the induced algebra structure on $\operatorname{Tor}_{*}^{Q}(R, k)$ provides for a classification of such local rings.

We describe the Macaulay2 package CodepthThree that implements an algorithm for classifying a local ring as above by computation of a few cohomological invariants.
\end{abstract}

INTRODUCTION AND NOTATION. Let $R$ be a commutative noetherian local ring with residue field $k$. Assume that $R$ has the form $Q / I$, where $Q$ is a regular local ring with maximal ideal $\mathfrak{n}$ and $I \subseteq \mathfrak{n}^{2}$. The embedding dimension of $R$ (and of $Q$ ) is denoted $e$. Let

$$
F=0 \longrightarrow F_{c} \longrightarrow \cdots \longrightarrow F_{1} \longrightarrow F_{0} \longrightarrow 0
$$

be a minimal free resolution of $R$ over $Q$. Set $d=\operatorname{depth} R$; the length $c$ of the resolution $F$ is

$$
c=\text { proj.dim } Q \text { depth } Q-\operatorname{depth}_{Q} R=e-d,
$$

by the Auslander-Buchsbaum formula, and one refers to this invariant as the codepth of $R$. In the following we assume that $c$ is at most 3. By a theorem of Buchsbaum and Eisenbud [Bruns and Herzog 1993, 3.4.3] the resolution $F$ carries a differential graded algebra structure, which induces a unique graded-commutative algebra structure on $A=\operatorname{Tor}_{*}^{Q}(R, k)$. The possible structures were identified by Weyman [1989] and by Avramov, Kustin, and Miller [Avramov et al. 1988]. According to the multiplicative structure on $A$, the ring $R$ belongs to exactly one of

Part of this work was done while the authors visited MSRI during the Commutative Algebra program in spring 2013. Christensen was partly supported by NSA grant H98230-11-0214.

MSC2010: primary 13P20; secondary 13D02, $13 \mathrm{H} 10$.

Keywords: local ring, Tor algebra. 
the classes designated $\mathbf{B}, \mathbf{C}(c), \mathbf{G}(r), \mathbf{H}(p, q), \mathbf{S}$, and $\mathbf{T}$. Here the parameters $p$, $q$, and $r$ are given by

$p=\operatorname{rank}_{k}\left(A_{1} \cdot A_{1}\right), \quad q=\operatorname{rank}_{k}\left(A_{1} \cdot A_{2}\right), \quad r=\operatorname{rank}_{k}\left(\delta: A_{2} \rightarrow \operatorname{Hom}_{k}\left(A_{1}, A_{3}\right)\right)$,

where $\delta$ is the canonical map. See [Avramov 2012; Avramov et al. 1988; Weyman 1989] for further background and details.

When, in the following, we talk about classification of a local ring $R$, we mean the classification according to the multiplicative structure on $A$. To describe the classification algorithm, we need a few more invariants of $R$. Set

$$
l=\operatorname{rank}_{Q} F_{1}-1 \quad \text { and } n=\operatorname{rank}_{Q} F_{c} ;
$$

the latter invariant is called the type of $R$. The Cohen-Macaulay defect of $R$ is $h=\operatorname{dim} R-d$. The Betti numbers $\beta_{i}$ and the Bass numbers $\mu_{i}$ record ranks of cohomology groups:

$$
\beta_{i}=\beta_{i}^{R}(k)=\operatorname{rank}_{k} \operatorname{Ext}_{R}^{i}(k, k) \quad \text { and } \quad \mu_{i}=\mu_{i}(R)=\operatorname{rank}_{k} \operatorname{Ext}_{R}^{i}(k, R) .
$$
The generating functions $\sum_{i=0}^{\infty} \beta_{i} t^{i}$ and $\sum_{i=0}^{\infty} \mu_{i} t^{i}$ are called the Poincaré series and
the Bass series of $R$.

THE ALGORITHM. For a local ring of codepth $c \leq 3$, the class together with the invariants $e, c, l$, and $n$ completely determine the Poincaré series and Bass series of $R$; see [Avramov 2012]. Conversely, one can determine the class of $R$ based on $e, c, l, n$, and a few Betti and Bass numbers; in the following we describe how.

Lemma 1. For a local ring $R$ of codepth 3 the invariants $p, q$, and $r$ are determined by e, $l, n, \beta_{2}, \beta_{3}, \beta_{4}$, and $\mu_{e-2}$ through the formulas

$$
\begin{aligned}
& p=n+l e+\beta_{2}-\beta_{3}+\left(\begin{array}{c}
e-1 \\
3
\end{array}\right), \\
& q=(n-p) e+l \beta_{2}+\beta_{3}-\beta_{4}+\left(\begin{array}{c}
e-1 \\
4
\end{array}\right), \\
& r=l+n-\mu_{e-2} .
\end{aligned}
$$

Proof. The Poincaré series of $R$ has the form

$$
\sum_{i=0}^{\infty} \beta_{i} t^{i}=\frac{(1+t)^{e-1}}{1-t-l t^{2}-(n-p) t^{3}+q t^{4}+\cdots}
$$

by [Avramov 2012, 2.1], and expansion of the rational function yields the expressions for $p$ and $q$. 
One has $d=e-3$ and the Bass series of $R$ has, also by [Avramov 2012, 2.1], the form

$$
\sum_{i=0}^{\infty} \mu_{i} t^{i}=t^{d} \frac{n+(l-r) t+\cdots}{1-t+\cdots}
$$

expansion of the rational function now yields the expression for $r$.

Proposition 2. A local ring $R$ of codepth 3 can be classified based on the invariants $e, h, l, n, \beta_{2}, \beta_{3}, \beta_{4}, \mu_{e-2}$, and $\mu_{e-1}$.

Proof. First recall that one has $h=0$ and $n=1$ if and only if $R$ is Gorenstein; see [Bruns and Herzog 1993, 3.2.10]. In this case $R$ is in class $\mathbf{C}(3)$ if $l=2$ and otherwise in the class $\mathbf{G}(l+1)$.

Assume now that $R$ is not Gorenstein. The invariants $p, q$, and $r$ can be computed from the formulas in Lemma 1. It remains to determine the class, which can be done by case analysis. Recall from [Avramov 2012, 1.3 and 3.1] that one has

\begin{tabular}{r|ccc} 
Class & $p$ & $q$ & $r$ \\
\hline $\mathbf{T}$ & 3 & 0 & 0 \\
$\mathbf{B}$ & 1 & 1 & 2 \\
$\mathbf{G}(r)[r \geq 2]$ & 0 & 1 & $r$ \\
$\mathbf{H}(p, q)$ & $p$ & $q$ & $q$
\end{tabular}

In case $q \geq 2$, the ring $R$ is in class $\mathbf{H}(p, q)$; for $q \leq 1$ the case analysis shifts to $p$. In case $p=0$, the distinction between the classes $\mathbf{G}(r)$ and $\mathbf{H}(0, q)$ is made by comparing $q$ and $r$; they are equal if and only if $R$ is in class $\mathbf{H}(0, q)$.

In case $p=1$, the distinction between the classes $\mathbf{B}$ and $\mathbf{H}(1, q)$ is made by comparing $q$ and $r$; they are equal if and only if $R$ is in class $\mathbf{H}(1, q)$.

In case $p=3$, the distinction between the classes $\mathbf{T}$ and $\mathbf{H}(3, q)$ is drawn by the invariant $\mu_{e-1}$. Recall the relation $d=e-3$; expansion of the expressions from [Avramov 2012, 2.1] yields $\mu_{e-1}=\mu_{e-2}+\ln -2$ if $R$ is in $\mathbf{T}$ and $\mu_{e-1}=\mu_{e-2}+\ln -3$ if $R$ is in $\mathbf{H}(3, q)$.

In all other cases, i.e., $p=2$ or $p \geq 4$, the ring $R$ is in class $\mathbf{H}(p, q)$.

Remark 3. One can also classify a local ring $R$ of codepth 3 based on the invariants $e, h, l, n, \beta_{2}, \ldots, \beta_{5}$, and $\mu_{e-2}$. In the case $p=3$ one then discriminates between the classes by looking at $\beta_{5}$, which is $\beta_{4}+l \beta_{3}+(n-3) \beta_{2}+\tau$, with $\tau=0$ if $R$ is in class $\mathbf{H}(3, q)$ and $\tau=1$ if $R$ is in class $\mathbf{T}$. However, it is not possible to classify $R$ based on Betti numbers alone. Indeed, rings in the classes $\mathbf{B}$ and $\mathbf{H}(1,1)$ have identical Poincaré series and so do rings in the classes $\mathbf{G}(r)$ and $\mathbf{H}(0,1)$. 
Remark 4. A local ring $R$ of codepth $c \leq 2$ can be classified based on the invariants $c, h$, and $n$. Indeed, if $c \leq 1$ then $R$ is a hypersurface; i.e., it belongs to class $\mathbf{C}(c)$. If $c=2$ then $R$ belongs to class $\mathbf{C}(2)$ if and only if it is Gorenstein ( $h=0$ and $n=1)$; otherwise it belongs to class $\mathbf{S}$.

Algorithm 5. From Remark 4 and the proof of Proposition 2, one gets the following algorithm that takes as input invariants of a local ring of codepth $c \leq 3$ and outputs its class.

Input: $c, e, h, l, n, \beta_{2}, \beta_{3}, \beta_{4}, \mu_{e-2}, \mu_{e-1}$.

- In case $c \leq 1$, set Class $=\mathbf{C}(c)$.

- In case $c=2$,

$\diamond \quad$ if $(h=0$ and $n=1)$ then set Class $=\mathbf{C}(2)$,

$\diamond \quad$ else set Class $=\mathbf{S}$.

- In case $c=3$,

$\diamond \quad$ if ( $h=0$ and $n=1)$ then set $r=l+1$,

- if $r=3$ : then set Class $=\mathbf{C}(3)$,

- $\quad$ else set Class $=\mathbf{G}(r)$;

$\diamond \quad$ else compute $p$ and $q$ :

- if ( $q \geq 2$ or $p=2$ or $p \geq 4)$ then set Class $=\mathbf{H}(p, q)$,

- else compute $r$ :

○ In case $p=0$,

- if $q=r$ then set Class $=\mathbf{H}(0, q)$,

- else set Class $=\mathbf{G}(r)$.

$\circ \quad$ In case $p=1$,

- if $q=r$ then set Class $=\mathbf{H}(1, q)$,

- else set Class $=\mathbf{B}$.

○ In case $p=3$,

- if $\mu_{e-1}=\mu_{e-2}+\ln -2$ then set Class $=\mathbf{T}$,

- else set Class $=\mathbf{H}(3, q)$.

Output: Class

Remark 6. Given a local ring $R=Q / I$ the invariants $e$ and $h$ can be computed from $R$, and $c, l$, and $n$ can be determined by computing a minimal free resolution of $R$ over $Q$. The Betti numbers $\beta_{2}, \beta_{3}, \beta_{4}$ one can get by computing the first five steps of a minimal free resolution $F$ of $k$ over $R$. Recall the relation $d=e-c$; the Bass numbers $\mu_{e-2}$ and $\mu_{e-1}$ one can get by computing the cohomology in degrees $d+1$ and $d+2$ of the dual complex $F^{*}=\operatorname{Hom}_{R}(F, R)$. For large values of $d$, this may not be feasible, but one can reduce $R$ modulo a regular sequence $\mathbf{x}=x_{1}, \ldots, x_{d}$ and obtain the Bass numbers as $\mu_{d+i}(R)=\mu_{i}(R /(\mathbf{x}))$; see [Bruns and Herzog 1993, 3.1.16]. 
The implementation. The Macaulay2 package CodepthThree implements Algorithm 5. The function torAlgClass takes as input a quotient $Q / I$ of a polynomial algebra, where $I$ is contained in the irrelevant maximal ideal $\mathfrak{N}$ of $Q$. It returns the class of the local ring $R$ obtained by localization of $Q / I$ at $\mathfrak{N}$. For example, the local ring obtained by localizing the quotient

$$
\mathbb{Q}[x, y, z] /\left(x y^{2}, x y z, y z^{2}, x^{4}-y^{3} z, x z^{3}-y^{4}\right)
$$

is in class $\mathbf{G}(2)$; see [Christensen and Veliche 2014]. Here is how it looks when one calls the function torAlgClass.

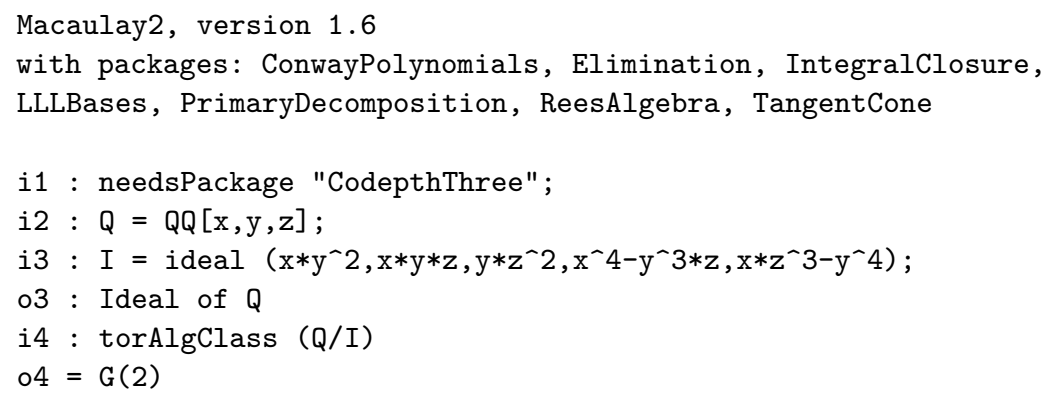

Underlying torAlgClass is the workhorse function torAlgData, which returns a hash table with the following data:

\begin{tabular}{|c|c|}
\hline Key & Value \\
\hline "c" & codepth of $R$ \\
\hline "e" & embedding dimension of $R$ \\
\hline "h" & Cohen-Macaulay defect of $R$ \\
\hline "m" & minimal number of generators of defining ideal of $R$ \\
\hline "n" & type of $R$ \\
\hline "Class" & (nonparametrized) class of $R$ \\
\hline "p" & $\begin{array}{l}\text { ('B', 'C', 'G', 'H', 'S', 'T', 'codepth }>3 \text { ', or 'zero ring') } \\
\text { rank of } A_{1} \cdot A_{1}\end{array}$ \\
\hline "q" & rank of $A_{1} \cdot A_{2}$ \\
\hline "r" & $\operatorname{rank}$ of $\delta: A_{2} \rightarrow \operatorname{Hom}_{k}\left(A_{1}, A_{3}\right)$ \\
\hline "PoincareSeries" & Poincaré series of $R$ \\
\hline "BassSeries" & Bass series of $R$ \\
\hline
\end{tabular}

In the example from above one gets:

$$
\begin{aligned}
& \text { i5 : } \operatorname{torAlgData}(\mathrm{Q} / \mathrm{I}) \\
& 2 \quad 34 \\
& 2+2 \mathrm{~T}-\mathrm{T}-\mathrm{T}+\mathrm{T} \\
& 05=\text { HashTable }\{\text { BassSeries }=>\text {--------------------- }\} \\
& 1-\mathrm{T}-4 \mathrm{~T}-2 \mathrm{~T}+\mathrm{T} \\
& c=>3
\end{aligned}
$$




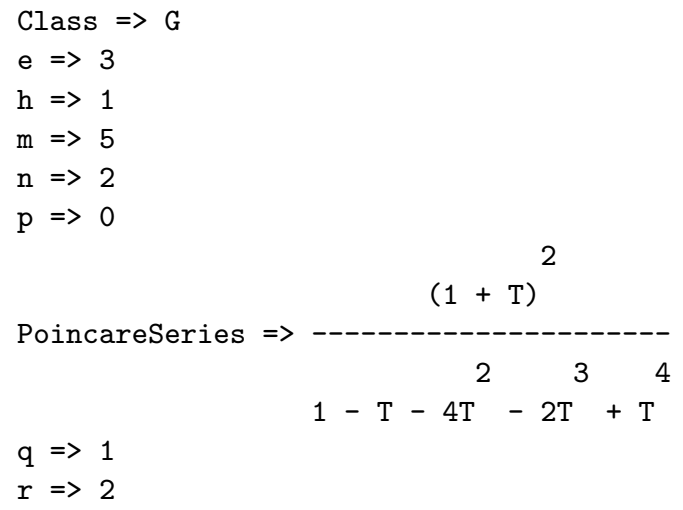

To facilitate extraction of data from the hash table, the package offers two functions torAlgDataList and torAlgDataPrint that take as input a quotient ring and a list of keys. In the example from above one gets:

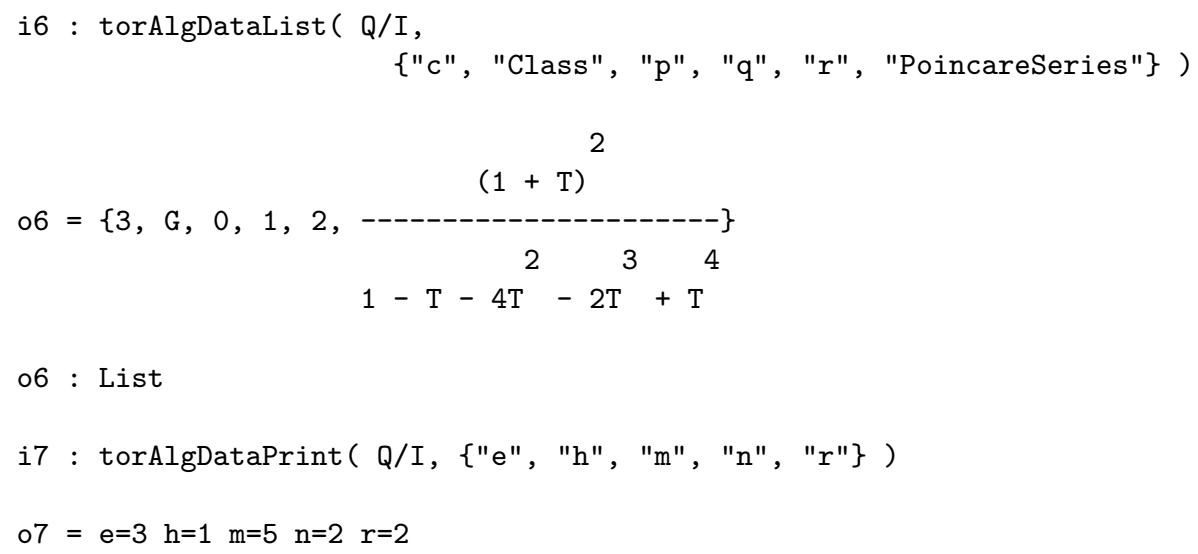

As discussed in Remark 6, the computation of Bass numbers may require a reduction modulo a regular sequence. In our implementation such a reduction is attempted if the embedding dimension of the local ring $R$ is more than 3 . The procedure involves random choices of ring elements, and hence it may fail. By default, up to 625 attempts are made, and one can change the number of attempts with the function setAttemptsAtGenericReduction. If none of the attempts are successful, then an error message is displayed:

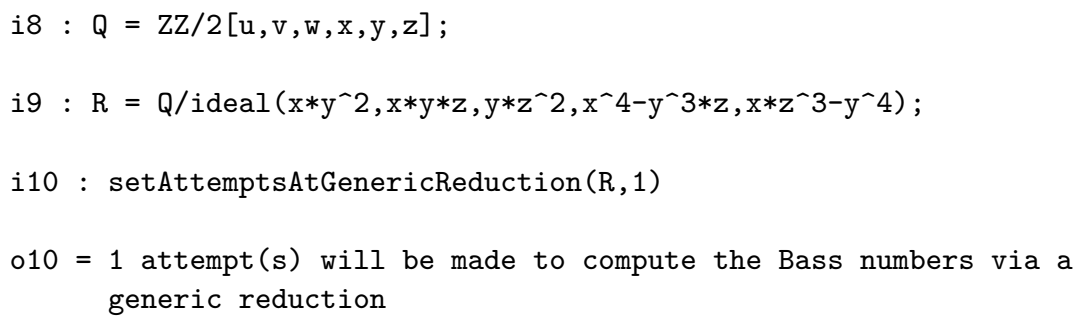




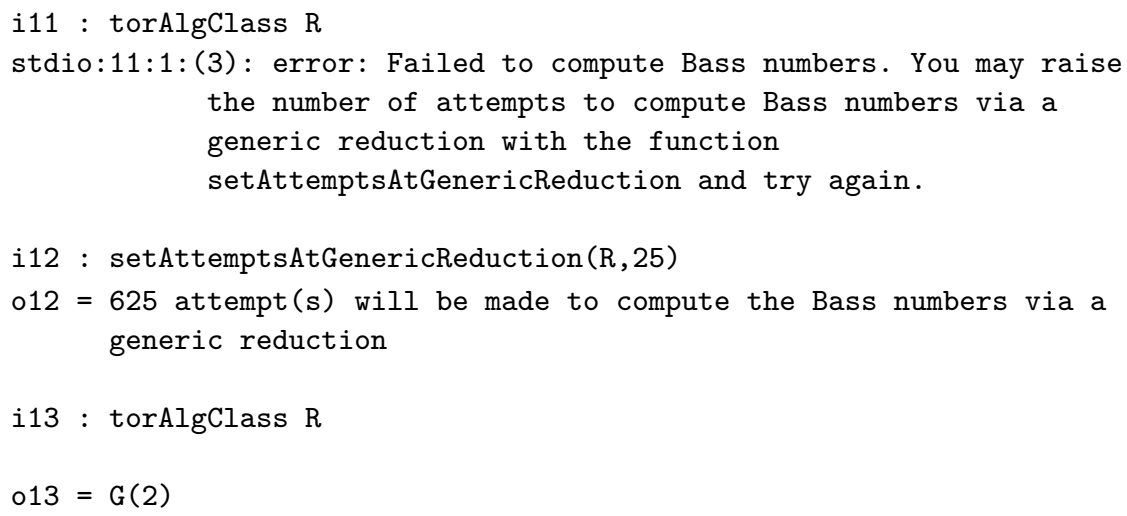

Notice that the maximal number of attempts is $n^{2}$, where $n$ is the value set with the function setAttemptsAtGenericReduction.

Notes. Given $Q / I$, our implementation of Algorithm 5 in torAlgData proceeds as follows.

(1) Check if a value is set for attemptsAtBassNumbers; if not use the default value 25 .

(2) Initialize the invariants of $R$ (the localization of $Q / I$ at the irrelevant maximal ideal) that are to be returned; see the table on page 5 .

(3) Handle the special case where the defining ideal $I$ or $Q / I$ is 0 . In all other cases, compute the invariants $c, e, h, m(=l+1)$, and $n$.

(4) If possible, classify $R$ based on $c, e, h, m$, and $n$. At this point the implementation deviates slightly from Algorithm 5, as it uses that all rings with $c=3$ and $h=2$ are of class $\mathbf{H}(0,0)$; see [Avramov 2012, 3.5].

(5) For rings not classified in steps (3) or (4), one has $c=3$; see Remark 4. Compute the Betti numbers $\beta_{2}, \beta_{3}$, and $\beta_{4}$, and with the formula from Lemma 1 , compute $p$ and $q$. If possible, classify $R$ based on these two invariants.

(6) For rings not classified in steps (3)-(5), compute the Bass numbers $\mu_{e-2}$ and $\mu_{e-1}$. If $d=e-3$ is positive, then the Bass numbers are computed via a reduction modulo a regular sequence of length $d$ as discussed above. Now, compute $r$ with the formula from Lemma 1 and classify $R$.

(7) The class of $R$ together with the invariants $c, l=m-1$, and $n$ determine its Bass and Poincaré series; see [Avramov 2012, 2.1].

If $I$ is homogeneous, then various invariants of $R$ can be determined directly from the graded ring $Q / I$. If $I$ is not homogeneous, and $R$ hence not graded, then functions from the package LocalRings are used. 


\section{REFERENCES.}

[Avramov 2012] L. L. Avramov, "A cohomological study of local rings of embedding codepth 3", $J$. Pure Appl. Algebra 216:11 (2012), 2489-2506. MR 2927181 Zbl 1259.13010

[Avramov et al. 1988] L. L. Avramov, A. R. Kustin, and M. Miller, "Poincaré series of modules over local rings of small embedding codepth or small linking number", J. Algebra 118:1 (1988), 162-204. MR 89k:13013 Zbl 0648.13008

[Bruns and Herzog 1993] W. Bruns and J. Herzog, Cohen-Macaulay rings, Cambridge Studies in Advanced Mathematics 39, Cambridge University Press, Cambridge, 1993. MR 95h:13020 Zbl 0788.13005

[Christensen and Veliche 2014] L. W. Christensen and O. Veliche, "Local rings of embedding codepth

3. Examples", Algebr. Represent. Theory 17:1 (2014), 121-135. MR 3160716 Zbl 06306457

[Weyman 1989] J. Weyman, "On the structure of free resolutions of length 3", J. Algebra 126:1 (1989), 1-33. MR 91g:13019 Zbl 0705.13008

RECEIVED: 16 Feb $2014 \quad$ REVISED: 11 Jul $2014 \quad$ ACCEPTED: 11 Jul 2014

LARS WINTHER CHRISTENSEN:

lars.w.christensen@ttu.edu

Department of Mathematics and Statistics, Texas Tech University, Lubbock, TX 79409,

United States

OANA VELICHE:

o.veliche@neu.edu

Department of Mathematics, Northeastern University, Boston, MA 02115, United States 